\title{
THE DESIGN OF A COMBINED OPTICAL DISC TESTER AND R- $\theta$ SCANNING LASER MICROSCOPE
}

\author{
N. A. E. HEYES, C. D. WRIGHT, W. W. CLEGG and J. ZHAO
}

The Manchester School of Engineering, University of Manchester, Manchester, M13 9PL, UK

\begin{abstract}
This paper describes the design of a novel, combined $\mathrm{R}-\theta$ scanning laser microscope and optical disk tester, which is to be used for the testing and evaluation of optical recording media. The instrument is capable of performing a range of standard read/write tests, whilst simultaneously yielding a microscopic image of the recorded bit structure with sub-micron resolution. A range of optical media can be investigated including magneto-optic, phase-change and write-once formats such as dye-polymer media.
\end{abstract} KEYWORDS: MAGNETO-OPTIC RECORDING, SCANNING LASER MICROSCOPY

\section{INTRODUCTION}

Magneto-optic scanning laser microscopes (SLMs) offer a means of imaging magnetic domain structures at high magnifications with significantly higher contrast than is obtainable in a conventional polarising microscope. Some of the capabilities of typical SLM designs are discussed in a companion paper[1]. In the current work we describe a novel SLM based instrument which combines the ability to record and replay magneto-optic domains at high headmedia speeds, together with the ability to image the recorded area with sub-micron resolution using a variety of contrast mechanisms. Available contrast modes include conventional non-magnetic contrast, polar Kerr contrast and magneto-optic phase contrast or edge detection[2,3] techniques. The system is also able to perform a wide range of standard recording tests on disk samples, including carrier to noise ratio measurements, pulse width determination and jitter characterisation. Imaging in the instrument is achieved by focusing a laser source to a diffraction limited spot on the surface of a rotating disk to obtain the line scan $(\theta)$ whilst moving the objective lens in the radial direction to obtain the field scan (R).

\section{OPTICAL SYSTEM DESIGN}

The optical layout of the instrument is shown in Fig. 1. To maximise the system's noise performance, the argon ion laser, which acts as the light source, is operated at high power (typically 1 Watt). Most of this power is then transmitted to a beam dump using the variable beamsplitter which is formed from the first polarising beamsplitter and halfwave plate in Fig. 1. In this way the laser operates at a point where fluctuations in its output are less than $0.2 \%$ of the total light output whilst the total power entering the instrument is maintained in the usual $5-20 \mathrm{~mW}$ range which is suitable for recording.

Since the instrument is designed to operate as an optical disk record and replay system as well as an R- $\theta$ scanning laser microscope, the light entering the instrument is split into two beams; one $\mathrm{S}$ polarised and one P polarised.

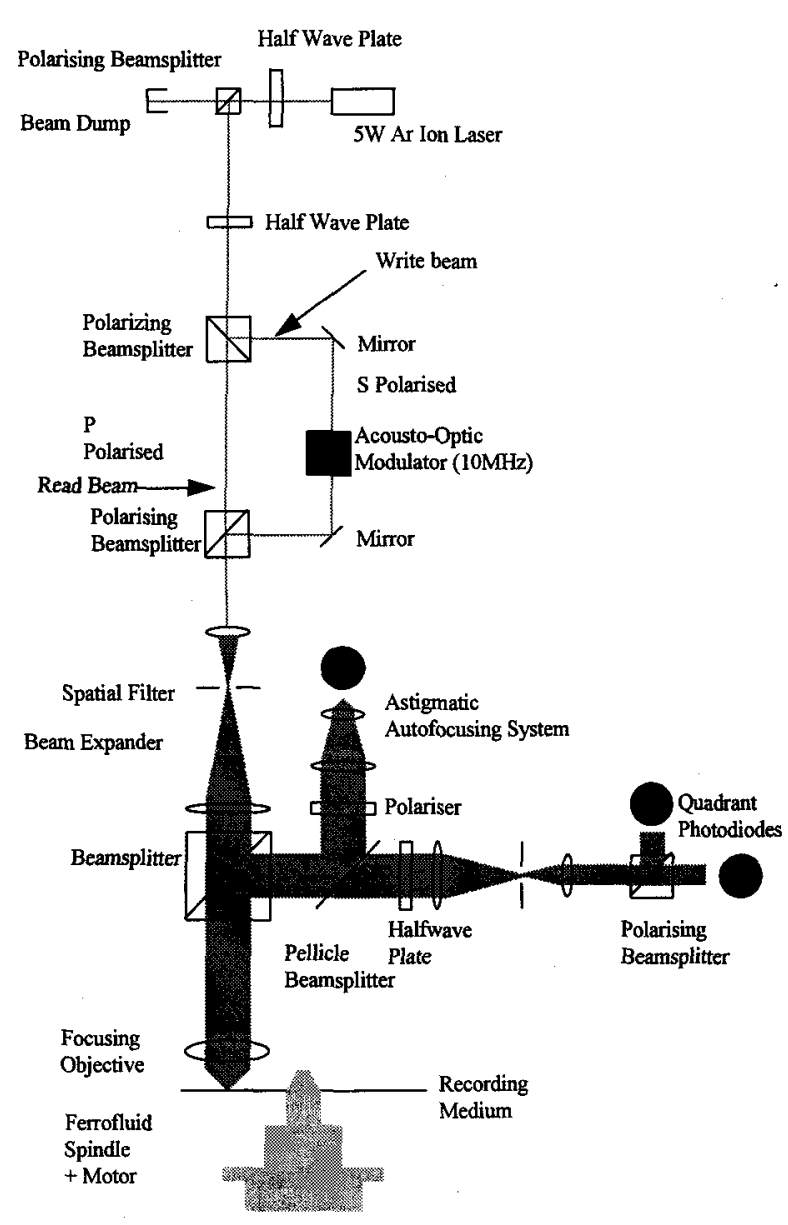

Fig. 1 Optical system layout

The intensities of these two beams are controlled by the position of a second halfwave plate. The lower powered, $P$ polarised read beam continuously illuminates the disk and is used to provide a feedback signal for maintaining the focus position of the objective lens, as well as for imaging the disk surface and for replaying recorded signals. The other, $\mathrm{S}$ polarised beam operates at higher powers and is high frequency modulated by a $10 \mathrm{MHz}$ acousto-optic modulator and is used for the recording of bit patterns. 
After recombination of the $S$ polarised write beam and the $P$ polarised read beam, the light passes through a spatial filter and beam expander. This results in an illuminating beam with a clean Gaussian profile. The spatial filter also acts as a stop for the zeroth order beam from the acousto-optic modulator, whilst the first order beam passes straight through. The incident light is focused, via the objective lens, onto a spinning disk sample that can rotate at speeds from 120 RPM up to 3000 RPM. The disk is mounted on a ferrofluid spindle which has a non-repeatable lateral and vertical run-out of less than $50 \mathrm{~nm}$. As this is well below the diffraction limit of the instrument, a track following servo is unnecessary. However, as it is impossible repeatably to mount and spin the disk in an entirely horizontal plane, a focusing servo is necessary and is provided by a standard astigmatic focus error detection and voice coil arrangement. The objective lens is mounted on a precision, piezo driven, linear translation stage. This enables the radial positioning of the lens to be controlled with a repeatability and a resolution of $0.05 \mu \mathrm{m}$. Circumferential position is determined to within $0.05 \mu \mathrm{m}$ via a quartz oscillator that phase-locks the signal from a rotary optical encoder which is mounted on the motor spindle.

The light reflected from the recording medium is split off by a non-polarising beamsplitter into the detection arm of the instrument. Ten per cent of this reflected light is sampled by the uncoated pellicle beamsplitter and after passing through a polariser, which rejects the $S$ polarised write beam, falls on a conventional astigmatic autofocusing system which generates a standard focus error signal. The remaining ninety per cent of the reflected light first passes through a halfwave plate that rotates the plane of polarisation of the reflected light such that it is at $45^{\circ}$ to the final polarising beamsplitter. The beam reducer ensures that the reflected light just covers the active areas of the quadrant diode detectors (a $1 \mathrm{~mm}$ diameter circle) and allows for confocal detection[4], if desired. The final polarising beamsplitter directs the $\mathrm{S}$ and $\mathrm{P}$ polarised components to the two detectors.

\section{SYSTEM OPERATION}

The use of quadrant detectors allows the instrument to produce the same wide range of imaging modes that are available on a more conventional X-Y scanning laser microscope, previously built in these laboratories, and described elsewhere[1,3,4,5].

The acquisition of images in the instrument is accomplished with a digital storage oscilloscope (DSO) which is connected via a high speed GPIB bus to an IBMtype PC computer (see Fig. 2). Up to four different, operator defined, combinations of the signals from the two quadrant detectors are formed using four identical channels of analogue electronics and the resulting signals are connected to the ' $Y$ ' inputs of the DSO. The speed of the

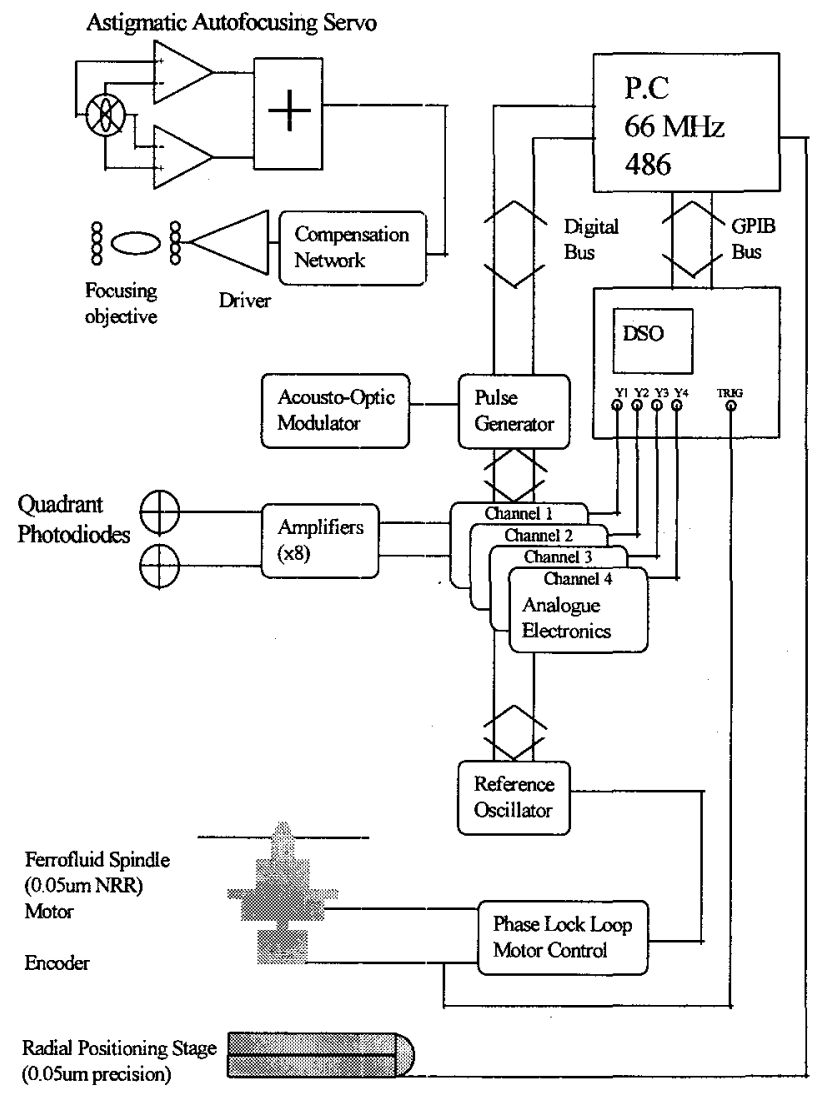

Fig. 2 Electronic System Layout

motor is maintained with a phase locked loop which phase locks, to a quartz oscillator, the output of a rotary encoder connected to the ferrofluid spindle. The resulting motor jitter is less than $0.005 \%$. Once per revolution of the disk a trigger signal is produced from the optical encoder which initiates the capture of a single line of the image. The start of the imaged area on the disk is determined by the angular position of the encoder which produces the trigger signal, the speed of the motor and the trigger delay set on the DSO. After the capture of one image line by the DSO the data is transferred to the PC over the GPIB bus where the data is translated into a grey scale image and displayed. After the display has been updated with the new image line, the radial position of the objective lens is increased and the DSO's trigger is rearmed. The actual distance moved by the objective lens determines the radial magnification of the final image whilst the magnification along each line of the image is determined by the timebase of the DSO. The system then waits for the disc to complete its revolution and generate the next trigger signal to initiate the capture of the next line of the image. In this way images of the surface of a disk may be obtained. As the DSO has four channels, four different signal combinations of the photodiode outputs may be formed and captured at the same time. Thus, up to four different images of the same area can be obtained in perfect registration with different image contrasts. For example, 
Fig. 3 illustrates two images of the same area. Fig. $3 a$ was obtained with polar Kerr contrast and shows an image of a magnetically recorded transition in a $\mathrm{P} t / \mathrm{Co} \mathrm{M}-\mathrm{O}$ recording film with the light and dark areas highlighting oppositely magnetised regions. The transition was recorded by first saturating the recording film by rotating the disk whilst moving an electromagnet radially across the disk's surface. The disk was then stopped and the field from the

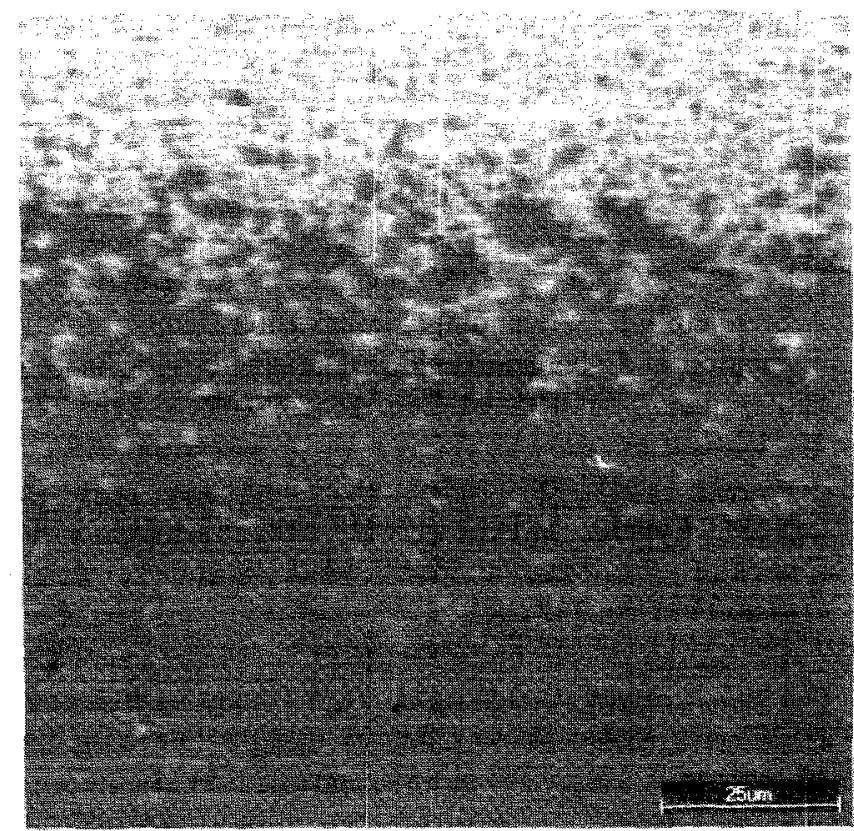

Fig. 3a R- $\theta$ SLM polar Kerr Image of a large scale magnetic transition in a $\mathrm{Pt} / \mathrm{Co}$ disc

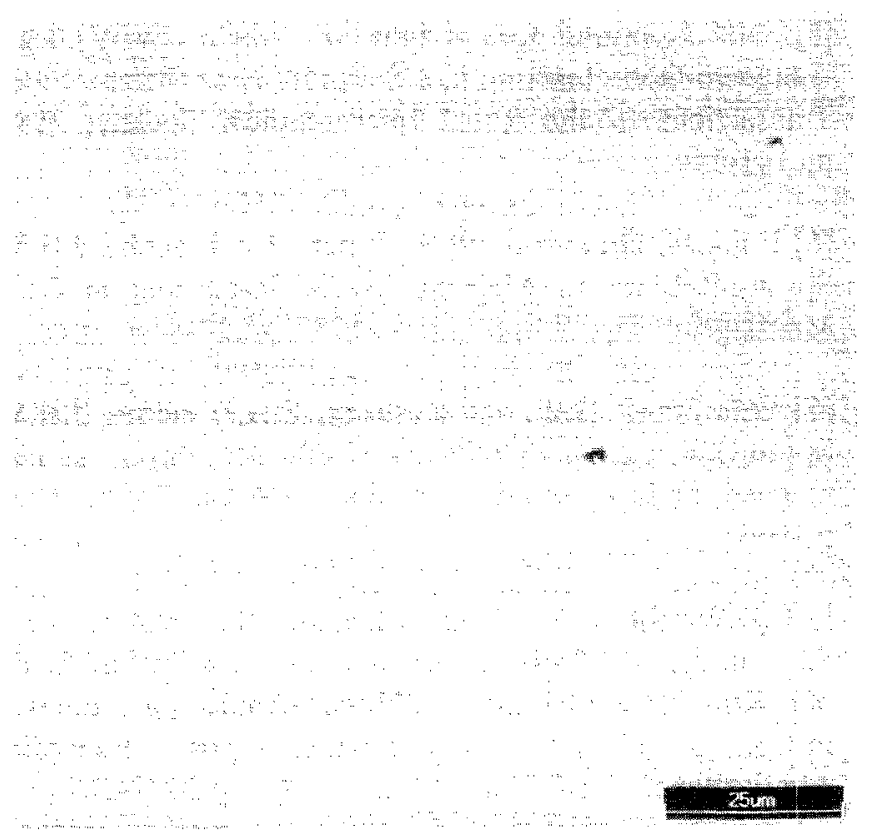

Fig. $3 b$ Reflected Light Image of the same area shown in Fig.3a electromagnet was reversed. Finally the electromagnet was switched off and the edge of the reversed area was imaged. Fig. 3b was obtained at the same time as Fig. 3a, but illustrates the total reflected light from this area of sample, and is therefore an ordinary, non-magnetic, surface contrast picture. Two pinhole surface defects are obvious in Fig. $3 \mathrm{~b}$ as dark areas, representing a reduction in the reflected light. In Fig. $3 a$ the pinholes are not so obvious and might be mistaken for magnetic contrast without the information provided by Fig. $3 \mathrm{~b}$. The recording film, which was composed of 16 periods of $4 \AA$ Co and $10 \AA \mathrm{Pt}$ on a glass substrate coated in a base layer of $100 \AA \mathrm{Pt}$ and deposited by electron beam evaporation at a substrate temperature of $200^{\circ} \mathrm{C}$, is imaged through the glass disk substrate. Vibrating sample magnetometer measurements on this film gave a room temperature coercivity of $1300 \mathrm{Oe}$.

\section{RECORDING TESTS}

To illustrate the use of the instrument as an optical disk tester, a $417 \mathrm{kHz}$ carrier was recorded by laser modulation. The carrier consisted of $400 \mathrm{~ns}$ write pulses separated by $2 \mu \mathrm{s}$. The carrier and noise levels were then measured at a bandwidth $\left(1 / \mathrm{nT}_{\mathrm{s}}\right)$ of $610 \mathrm{~Hz}$ at a linear velocity of $1.5 \mathrm{~m} / \mathrm{s}$. The field applied during recording was 100 Oe and the power of the laser during reading was $3 \mathrm{~mW}$. Fig. 4 illustrates the change in carrier and noise levels as the recording power is increased. The write laser power was in excess of $13 \mathrm{~mW}$. The high laser power required for recording on this film is probably due to the increased Curie temperatures of $\mathrm{Pt} / \mathrm{Co}$ multilayers where the Pt thickness is around $10 \AA[6]$. The Pt underlayer has also been shown to result in a recording film with a more particulate microstructure and a higher coercivity and would also act as a heat sink during recording[7,8]. The increase in carrier power shown in Fig. 4 is due in part to the increasing domain size at high write laser powers. This increasing bit

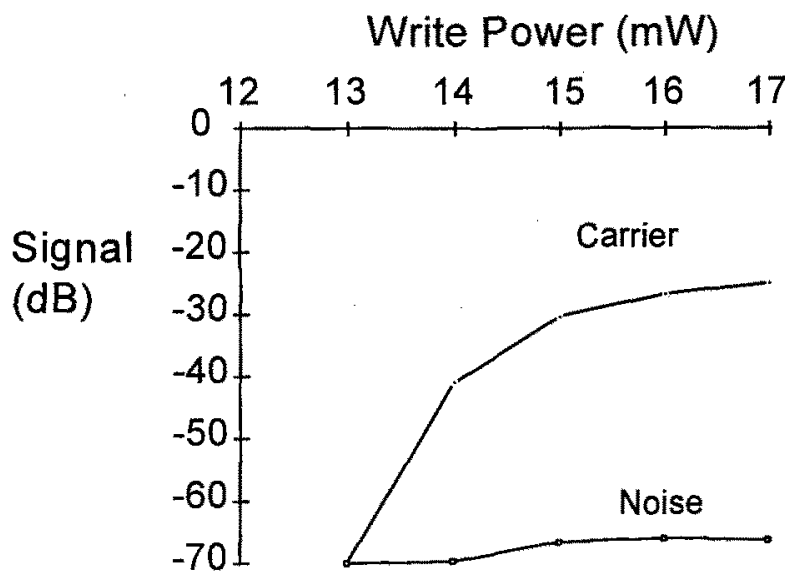

Fig. 4 Carrier and Noise Levels 
size is shown in Fig. 5, where full width half maximum read pulse measurements, averaged over fifty domains, are shown. A polar Kerr image of the disk area recorded for the CNR measurements is shown in Fig. 6. The image shows 12 data tracks, of which there are three tracks written at power levels of $17 \mathrm{~mW}$ (top of image), $16 \mathrm{~mW}, 15 \mathrm{~mW}$ and $14 \mathrm{~mW}$ (bottom of image).

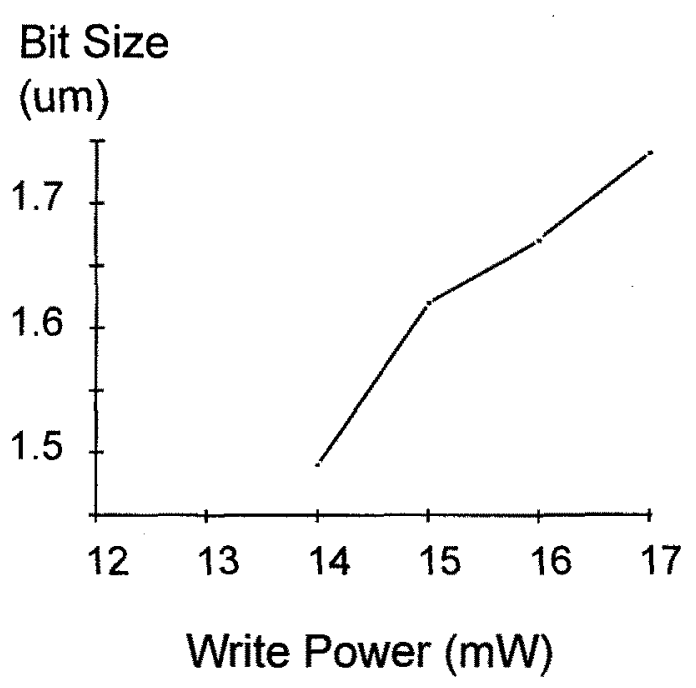

Fig. 5 Bit size Vs. Write Power

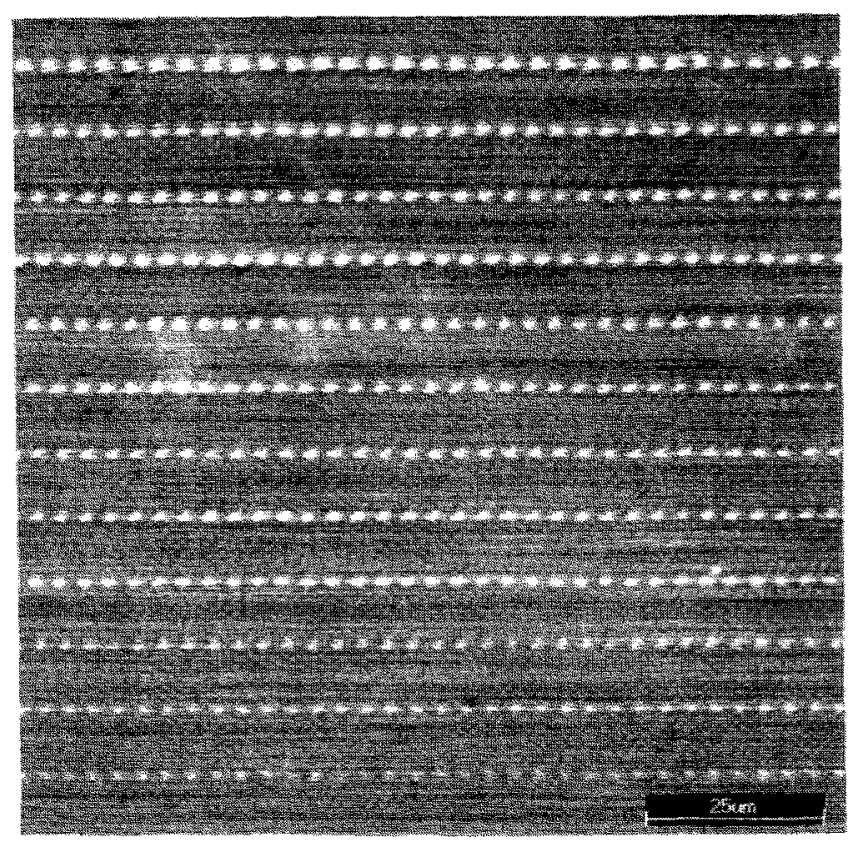

Fig. 6 R- $\theta$ SLM polar Kerr image of the recorded data tracks

\section{SUMMARY}

The design and operation of a novel combined R- $\theta$ scanning laser microscope and optical disk spin stand has been described in detail. Initial experimental recording results obtained from this instrument have been presented and indicate the usefulness of the instrument for imaging the recorded domain structures and for characterising magnetooptic recording media.

\section{ACKNOWLEDGEMENTS}

The authors would like to acknowledge the SERC for supporting this work, Dr E.W.Hill for providing assistance with the e-beam deposition system and Kate Hill of Pilkington Micronics, Clwyd for providing the glass disk substrates.

\section{REFERENCES}

[1] C.D.Wright, W.W.Clegg, N.A.E.Heyes and A.Boudjemline: "Scanning laser microscopy for the evaluation and characterisation of magneto-optic materials and systems", MORIS 94 Conference, Tokyo, Sept 27-29, 1994

[2] R.T.Lynch, M.D.Levenson and S.M.Tan: "Edge Detection for Magnetooptical data Storage", Applied Optics, 1991, pp.232-252

[3] N.A.E.Heyes, C.D.Wright, W.W.Clegg; "Observations of Magneto-Optic Phase Contrast using a Scanning Laser Microscope", J.Appl.Phys., 1991, pp.5322-5324

[4] C.D.Wright, N.A.E.Heyes: "A Comparison of Various Readout Techniques in Magneto-Optic Recording", IEEE Trans. Magn., 1991, pp. 5127-5129

[5] N.A.E.Heyes, C.D.Wright, E.W.Hill, W.W.Clegg: "Magneto-Optic Imaging in a Scanning Laser Microscope", Transactions of the Royal Microscopical Society, 1990, pp. 141-144

[6] F.J.A.M. Greidanus, W.B. Zeper, B.A.J.Jacobs, J.H.M. Spruit, P.F.Carcia: "Magneto-Optical Recording in $\mathrm{Co} / \mathrm{Pt}$ Multilayers", Jap. J. Appl. Phys., 1989, pp.37-44

[7] W.B Zeper, H.W. van Kesteren, B.A.J. Jacobs, J.H.M. Spruit, P.F. Carcia: "Co/Pt and $\mathrm{Co} / \mathrm{Pd}$ Multilayers as new magneto-optical recording media", J.Appl. Phys., 1991, p.4966

[8]C.D.Wright, W.W.Clegg, A.Boudjemline, N.A.E.Heyes, P.J.Grundy, R.J.Pollard, S.J.Greaves, A.K.Petford-Long, Y.H.Kim and J.P.Jakubovics: "Micro-Annealing Studies of Pt/Co Multilayers for Magneto-Optic Recording Applications", IEEE Trans. Magn., 1992, pp.2671-2673 